\author{
Case Study
}

\title{
AYURVEDIC SUCCESSFUL MANAGEMENT OF NODULAR EPISCLERITIS WITH PROLONGED PERIOD FOLLOW UP-A CASE REPORT
}

\section{Shantala Priyadarshini' ${ }^{*}$, Pannaga N Terragundi², Pooja Gangadkar ${ }^{3}$, Priyanka}

${ }^{*}$ Director and Senior Consultant, ${ }^{4}$ Research Associate, Dr. Vijay's Ayurveda Research \& Therapy centre, Mysore, India.

2,3PG Scholar, Sri Dharmasthala Manjunatheshwara Ayurvedic Medical College, Bengaluru, Karnataka.

\begin{tabular}{l} 
Article info \\
Article History: \\
Received: 01-09-2021 \\
Revised : 12-09-2021 \\
Accepted: 26-09-2021 \\
Published: 16-10-2021 \\
\hline KEYWORDS: \\
Nodular \\
Episcleritis, \\
Prolonged Period \\
Follow Up, \\
Mahayogaraja \\
guggulu, Balarista.
\end{tabular}

ABSTRACT
Episcleritis, an acute unilateral or bilateral inflammation, usually idiopathic or autoimmune
condition with or without underlying systemic condition is a troublesome manifestation of
red eye reported. It manifests as red or pinkeye, varied mild pain to foreign body sensation,
tender discomfort on touch, commonly recurs. As an acute condition patient seeks medical
assistance. Ayurveda recognised this either as Sirajala, Siraharsha, Sirajapidika and a
Shuklabhagaroga. Here a young lady with painful repeated manifestation of episcleritis is
reported seeking Ayurveda for holistic management of the condition after repeatedly trying
other systems in vain. Patient was treated with suitable Shodhana, Kriyakalpa followed by
necessary Dinacharya and Rasayana ensuring non recurrence; Deepan pachana, Anulomana,
Kriyakalpa, Rasyana chosen were all Chakshushyadravyas. To add up to synchronize the
effect of yoga therapies advised, to achieve non recurrence of symptoms.
Trikatu, Trivrt, Triphala, Yashtimadhu, Madhu, Ghrta known Chakshushyadravya having
vitamins and trace elements known to be highly beneficial to the eye and all sense organs
was advocated, Kriyakalpa play a major role in immediate mitigation of symptoms and also
prevents recurrence. Seka is most accepted Kriyakapla for enhancing quick relief, non
recurrence, cost effective Ayurveda management reported after a prolonged period of
follow up for a year. Yoga therapy included Suryanamaskar, Anulomaviloma pranayama and
Trataka, Ayurveda and yoga when added to management ensured non recurrence.

\section{INTRODUCTION}

Episcleritis, idiopathic and self limiting or as an autoimmune condition. Any underlying general systemic conditions as causative factor is reported and prevalent more in female adults. Also has types viz, nodular, diffuse and simple. manifest in single or both eyes, could be with no symptoms and tender and painful manifestation. ${ }^{[1-3]}$.

Benign inflammation of the conjunctival and superficial episcleral vascular plexi is also known as subconjunctivitis, phlegmatous conjunctivitis, episcleritis periodica fugax.

\begin{tabular}{|l|l|}
\hline \multicolumn{3}{|c|}{ Access this article online } \\
\hline Quick Response Code & \begin{tabular}{l} 
https://doi.org/10.47070/ijapr.v9i9.2010 \\
\hline
\end{tabular} \\
\hline
\end{tabular}

In Ayurveda, Episcleritis is similar to a condition called as Sirajala, a type of Shuklagathanetraroga (Disease of sclera) as explained by Acarya Susruta. It is a Raktaja Chedana Sadhyavyadhi (curable through excision). It presents with symptoms like Jalabha (network of vessels), Katina sira Mahan (tough raised vessels). The general line of management for Sirajala is Bheshaja (Abhishyandavat- similarly treated like Conjunctivitis management) as a first line of treatment, Abhishyandha as it's said to be the Purvarupa of most eye diseases. If not cured by Bheshaja then Chedana (excision) is indicated[4].

It is usually benign but few cases recur so frequently needing continued medications and may result in complications like various uveitis, visual disturbances, glaucoma and advance to cataracts. Medications include non -steroidal anti inflammatory drugs to relieve pain and other symptoms but to stop recurrence Ayurveda can be of immense assistance 
with various herbal formulations. Many stalwarts shalakii s have reported case studies. ${ }^{[5-9]}$

\section{Case Report}

A young lady aged 18 years presented with severe ciliary congestion in both eyes, elevated bulbar conjunctiva and complained of painful gritty sensation, headache, intermittent mild fever and malaise. Medical history as below made the patient turn towards Ayurveda management. Medical history -Patient had similar episodes of acute pain, ciliary congestion and was on the following medications. $1.800 \mathrm{mg}$ ibuprofen three times daily and after a week was advised flurbiprofen $100 \mathrm{mg}$ three times daily-relief from pain and redness was evident. Patient developed nausea and stomach pain after dosage was reduced. Redness cleared and the patient developed the above side effects. So the medicines were stopped. P, allergic conjunctivitis, visual problems and was treated in vein. Patient was advised topical ophthalmic $0.5 \%$ prednisolone. Was diagnosed as episcleritis. 5th episode 2 weeks after medications have been stopped. Symptoms recurred. After 15 days of stopping medicines, gradually symptoms recurred. These symptoms kept repeating. So blood tests and chest Xray was advised. Negative Mantoux test, raised ESR. So was advised ATT- Isoniazid (INH), Rifampicin (RIF), Ethambutol (EMB), pyrazinamide (PZA). Patient stopped all medications and opted for holistic management. Repeated monthly episodes.

Table 1: Symptoms Scale Admission, Discharge, Follow up

\begin{tabular}{|l|l|l|l|}
\hline Symptoms & $\begin{array}{l}\text { Scale Before } \\
\text { Management admission }\end{array}$ & $\begin{array}{l}\text { Scale after management } \\
\text { day of discharge }\end{array}$ & $\begin{array}{l}\text { Scale after management 9 } \\
\text { months after discharge }\end{array}$ \\
\hline Redness & ++++++ & ------------- & ---------- \\
\hline Tender & +++ & + & -------- \\
\hline Lacrimation & ------- & --- & --- \\
\hline FB sensation & +++ & Nil & Nil \\
\hline Headache & ++++ & occasional & Rarely \\
\hline Photophobia & ++ & Nil & Nil \\
\hline Dryness & ++++++ & + & + \\
\hline
\end{tabular}

Differential Diagnosis- conjunctivitis, phlyctenular conjunctivitis, scleritis were considered. DiagnosisRecurrent episcleritis with mild RA Assessment Slit lamp examination- Sectorial slightly elevated bulbar conjunctiva, engorged episcleral vessels bright red, no foreign body present, no blepharitis or trichiasis. Cornea and lids normal Dryness checked using Schirmer strip indicates dryness Objective assessment visual analog scale (VAS) Symptom Universal pain assessment scale 0 --10 scale patient assessment ${ }^{[10]}$ was adopted. Activity tolerance scale --No pain, Can be ignored, Interferes with task, Interferes with concentration, Interferes with basic needs, bed rest required. Assessment in Dry Eye[11] Schirmer strip indicates dryness Shushkatha (mild dryness -greater than 15) Shushakshipaka (moderately dry eye) 10 to 15

Table 2: Symptoms Reduction Scale- During, After management

\begin{tabular}{|l|l|l|l|}
\hline Symptoms & Before & During management & After management \\
\hline Subjective & & & \\
\hline Redness & ++++++ & +++ & ---- \\
\hline Pain & +++ & + & -- \\
\hline Lacrimation & + & + & - \\
\hline FB sensation & +++++ & ++ & - \\
\hline Headache & +++ & + & - \\
\hline Photophobia & + & + & - \\
\hline Objective & & & \\
\hline Dryness & Moderate 12 & Mild 18 & Mild 18 \\
\hline Tenderness & ++++ & ++ & - \\
\hline
\end{tabular}


Int. J. Ayur. Pharma Research, 2021;9(9):74-79

Table 3: Balarista- Balarishta ref Bhaishajya Ratnavali Vatavyadhi pp.567-572

\begin{tabular}{|l|l|l|l|}
\hline Ingredient & Quantity & Ingredient & Quantity \\
\hline Bala & 1 Tula & Eranda & 2 Pala \\
\hline Ashwagandha & 1 Pala & Rasna & 1 Pala \\
\hline Guda & 3 Pala & Ela & 1 Pala \\
\hline Jala & 4 Drona & Prasarini & 1 Pala \\
\hline Dhataki Pushpa & 16 Pala & Usheera & 1 Pala \\
\hline Payasya & 2 Pala & Gokshura & 1 Pala \\
\hline
\end{tabular}

Table 4: Mahayogaraja guggulu- Sharangdhara Samhita Madhyamakhanda 7/56-69

\begin{tabular}{|l|l|l|l|}
\hline Ingredients & Quantity & Ingredients & Quantity \\
\hline $\begin{array}{l}\text { Nagaradi (20) } \\
\text { Prakshepaka }\end{array}$ & 20 Parts & Vanga Bhasma & 1 Pala \\
\hline Amalaki & 40 Parts & Raupya Bhasma & 1 Pala \\
\hline Haritaki & 40 Parts & Naga Bhasma & 1 Pala \\
\hline Vibhitaki & 40 Parts & Loha Bhasma & 1 Pala \\
\hline Suddha Guggulu & 140 Parts & Abhraka Bhasma & 1 Pala \\
\hline Guda & QS & Mandura Bhasma & 1 Pala \\
\hline Gritha & QS & Rasa Sindura & 1 Pala \\
\hline
\end{tabular}

Table 5: Timeline Management

\begin{tabular}{|c|c|c|}
\hline Date & Management & Details \\
\hline 12.06 .2018 & $\begin{array}{l}\text { Deepana, Pachanaanulomana } \\
\text { 3days }\end{array}$ & $\begin{array}{l}\text { Trikatu } 1 / 4 \text { teaspoon + honey twice a day } \\
\text { Triphala churna } 1 / 4 \text { teaspoon in hot water bedtime } \\
\text { administered. }\end{array}$ \\
\hline 15.06.2018 & $\begin{array}{l}\text { Pratimarshanasyaanutaila } \\
\text { Seka twice a day }\end{array}$ & $\begin{array}{l}\text { Twice a day for } 1 \text { week continued once a day for a } \\
\text { month. } \\
20 \text { mins Seka - Yastimadhu churna+ Sariva churna } \\
+ \text { Ksheera + Jatpatra Swarasa not warm continued } \\
\text { full time }\end{array}$ \\
\hline 22.06.2018 & $\begin{array}{l}\text { Shoolashotaharabidalaka twice } \\
\text { a day for a } 6 \text { weeks Charaka } \\
\text { Trimarmeeya chikitsa }\end{array}$ & $\begin{array}{l}\text { Nimbhapatra paste } 1 / 2 \text { teaspoon }+ \text { Nagara churna } 1 / 2 \\
\text { teaspoon }+3 \text { grains Saindavalavan continued full } \\
\text { time }\end{array}$ \\
\hline 12.01.2019 & $\begin{array}{l}\text { Oral medication as in table } \\
\text { as in table below }\end{array}$ & $\begin{array}{l}\text { Mahayogarajaguggulu } 500 \mathrm{gm} \text { crushed added to } \\
10 \mathrm{ml} \text { Balarishta+ equal water twice a day after } \\
\text { food for } 6 \text { months }\end{array}$ \\
\hline 21.06.2019 & $\begin{array}{l}\text { Trataka, Yoga, Pranayama- } \\
\text { whole } 4 \text { months daily once }\end{array}$ & $\begin{array}{l}\text { Patient was taught ghee lamp Jyotitratak } \\
\text { Suyanamskara, Sarvangasana, Shavasana } \\
\text { Anulomaviloma pranayama }\end{array}$ \\
\hline 21.06.2019 & $\begin{array}{l}\text { Keshamasianajana prepared as } \\
\text { per Vagbhata in Shuskashipaka }\end{array}$ & $\begin{array}{l}\text { Was started after } 1 \text { week, once a day. Advised to } \\
\text { be continued for } 6 \text { months }\end{array}$ \\
\hline
\end{tabular}




\section{Day of Admission (12.06.2018)}
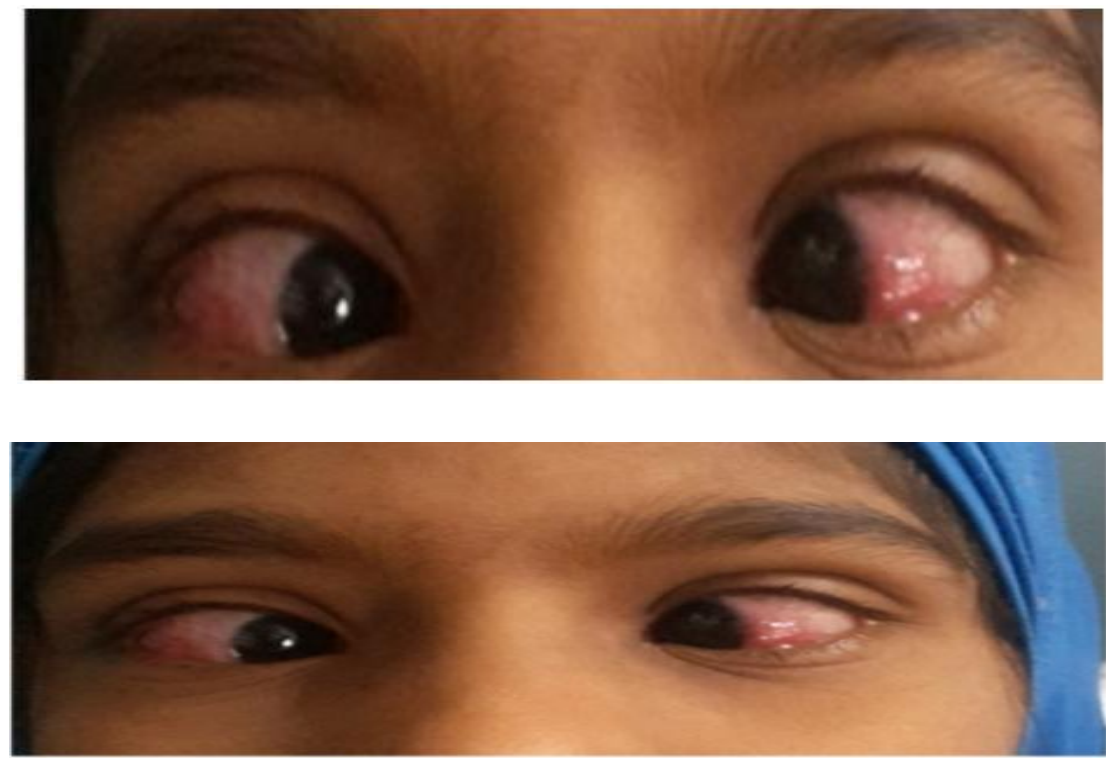

After Deepana pachana anulomana (15.06.2018)

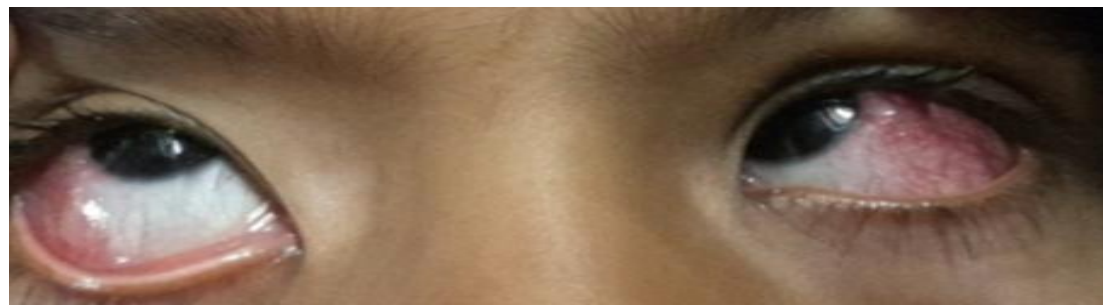

After Seka, Bidalaka (22.06.2018)

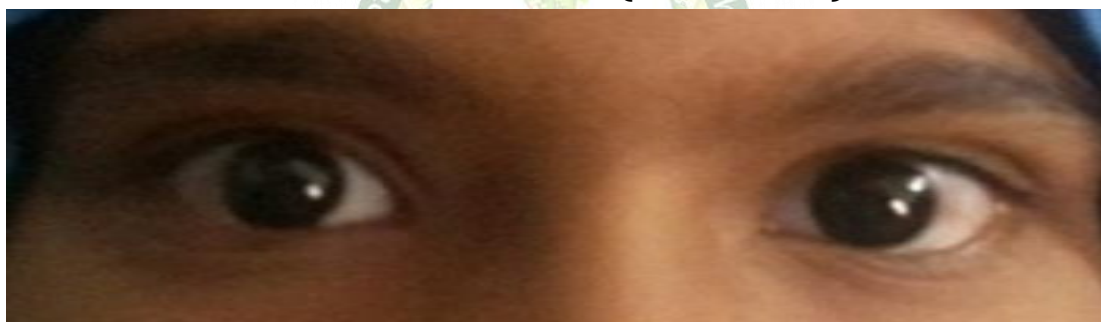

Just on the day of discharge $(22.07 .2018)$

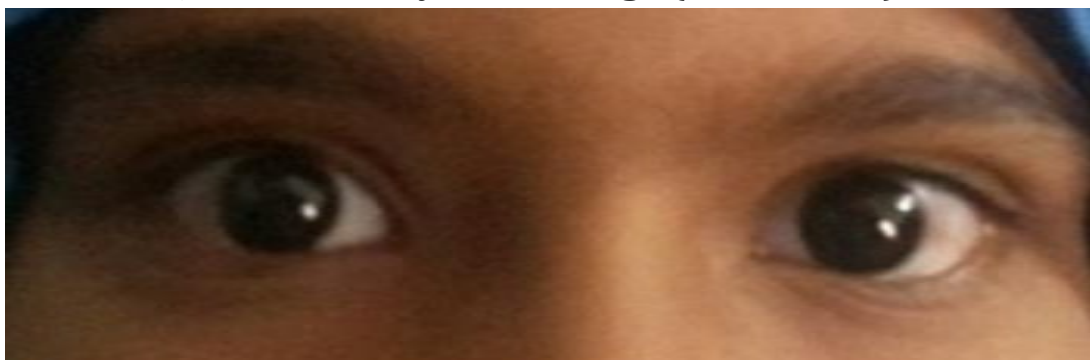

6months follow up after discharge - no recurrence (12.01.2019)

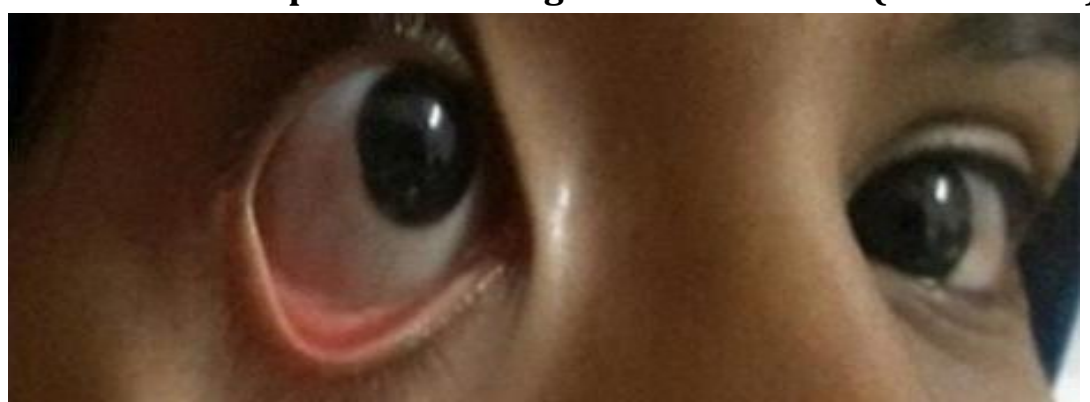

9months- No Recurrence (21.06.2019) 


\section{RESULTS}

Patient got total relief from pain, tenderness, photophobia and redness in 4 weeks but dryness though reduced was still not totally normal so medications were continued for almost 6months and when no recurrence was noted all medications were stopped and patient follow up next three months was uneventful as no recurrence was reported.

\section{DISCUSSION}

Normal status of Agni is very important so Deepana, Pachana and Anulomana was initiated in the beginning as Ayurveda believes all conditions start due to errors in metabolism.[26]

Seka, Bidalaka have been adopted in many eye conditions to mitigate pain, inflammation and here too such Kriyakalpas have been found beneficial.

Anutaila is best for application as Pratimarshanasya as it is also known as antiinflammatory, effective in Urdwajatragatarogas it is beneficial for daily application.[27]

Management included anti-inflammatory therapies, immunomodulators and it was holistic management to ensure no recurrence.

Ophthalmic Uses and Indications: Neem Netrarbadhahara (analgesic ophthalmic action), Netrakanduhara (anti-allergic action), Netra sotha hara (indicated in ocular inflammations) Netrya (conducive in ocular conditions) (Srikanth; N. 2000).

Holistic management includes oral medication like Mahayogaraja guggulu 500gm crushed and dissolved in Balarista $20 \mathrm{ml}$ with equal water to be taken after meals as it is best absorbed and acts faster.

\section{REFERENCES}

1. Schonberg S, Stokkermans TJ. Episcleritis. [Updated 2021 Aug 11]. In: Stat Pearls [Internet]. Treasure Island (FL): Stat Pearls Publishing; 2021 Jan-. PMID: 30521217.

2. https://www.ncbi.nlm.nih.gov/books/NBK534796

3. Sainz de la Maza M, Molina N, Gonzalez-Gonzalez LA, Doctor PP, Tauber J, Foster CS. Clinical characteristics of a large cohort of patients with scleritis and episcleritis. Ophthalmology. 2012 Jan; 119(1):43-50.

4. Watson PG, Hayreh SS. Scleritis and episcleritis. $\mathrm{Br}$ J Ophthalmol. 1976 Mar; 60(3): 163-91. [PMC free article] [PubMed]

5. Murthy Srikantha K R. Illustrated Susruta Samhita Uttara tantra15/21.Varanasi: Chaukhamba Orientalia; 2010.

6. Namboothiri P. Sreekanth, et al: Management of Scleritis Through Ayurveda- A Case Report. International Ayurvedic Medical Journal \{online\} 2019 \{cited February, 2019\} Available from: http://www.iamj.in/posts/images/upload/291_29 5.pdf Martin Grueterich, Edgar Espana, EikiGotoClin Ophthalmol. 2008 Mar; 2(1): 31-55. doi: 10.2147/opth.s1496 PMCID: PMC2698717

7. Schiffman RM, Christianson MD, Jacobsen G, et al. Reliability and validity of the Ocular Surface Disease Index. Arch Ophthalmol. 2000; 118: 615621.

8. Vardhan P, Dhiman KS, Sharma SK. Shimla: HPU; A clinical study on the effect of Keshanjana and Parisheka in the management of Sushkakshipaka w.s.r. to Dry Eye Syndrome; p. 2005.

9. Dhiman KS, Vyas M, Prajapati PK. Standard operational procedures of Keshanjana. Int J Ayu Med. 2012; 3:152-5.

10. https://onlinelibrary.wiley.com/doi/epdf/10.1111 /aos.14484

11. Choudhury ry C, Manjusha R, Bavalatti N. A clinical success in Sirapidika (episcleritis) with Saindhavadi Bidalaka yoga and oral medications A Case Report. In t. J. AYUSH CaRe. 2019; 3(3): 197 $-200$

12. Priyadarshini ST, Remitha K K, Priyanka S. Ayurvedic management of co-infection of Herpes zoster ophthalmicus in COVID-19 patient: A case report. J Ayurveda Case Rep 2021; 4: 44-9

13. Clinical Observation of Nimba (Azadirachta Indica A. Juss.) And Sirisha (Albizia Lebbeck Benth.) In Simple Epidemic Viral Conjunctivitis.

14. S, Lokeshwar T, Mukesh B \& Vishnu B, Review on neem (Azadirachta indica) Thousand problems one solution. Int Res J Pharm, 2 (2011) 97.

15. Topical Ginger Treatment With a Compressor Patch for Osteoarthritis Symptoms

16. Tessa Therkleson J Holist Nurs. 2014 Sep; 32(3): 173-182. doi: 10.1177/0898010113512182

17. PMCID: PMC4230973Afzal M al-hadidi D, Menon M, Pesek J, Dhami MS (2011). Ginger: An Ethnomedical, Chemical and Pharmacological Review. Drug Interact. 18:159-190.

18. Muzaffer Alam, K.K Shanmuga Dasan, Susan Thomas, Joy Suganthan, Anti-Inflammatory Potential of Balarishta and Dhanvantara Gutika In Albino Rats, Anc Sci Life. 1998 Apr-Jun; 17(4): 305-312. PMCID: PMC3331117

19. H.K Amarnath: Ayurvedic Management of Sirajala (Episcleritis)- A case report; ayurpub; IV(4): 13051309

20. Choudhury, C., Rajagopala, M., \& Bavalatti, N. (2019, October 3). A clinical success in Sirapidika (episcleritis) with Saindhavadi Bidalaka yoga and oral medications - A Case Report. International Journal of AYUSH Case Reports, 3(3), 197- 
200.https://doi.org/https://doi.org/10.52482/ijac are.v3i3.95

21. Shital S. Bolkuntwar: A Case Study - Management of Episcleritis with Jalaukavacharan. International Ayurvedic Medical Journal \{online\} 2017 \{cited September, $\quad 2017\} \quad$ Available from:http://www.iamj.in/posts/images/upload/3 649_3655.pdf

22. Anju D, Pushpa RP, Ashwini MJ. Ayurvedic management of Episcleritis (Sira Pidaka)- A Case Study. J AyurvedaIntegr Med Sci 2017; 1:254257.The challenge of dry eye diagnosis Giacomo Savini, Pinita Prabhawasat, Takashi Kojima.

23. G. Gopinathan, Kartar Singh Dhiman, R. Manjusha,A clinical study to evaluate the efficacy of Trataka Yoga Kriya and eye exercises (nonpharmocological methods) in the management of Timira (Ammetropia and Presbyopia) Ayu. 2012 Oct-Dec; 33(4): 543-546. doi: 10.4103/09748520.110534PMCID: PMC3665208

24. Gopal KS, Bhatnagar OP, Subramanian N, Nishith SD. Effect of yogasanas and pranayamas on BP, pulse rate and some respiratory functions. Indian J PhysiolPharmacol. 1973; 17: 273-6.

25. Bhutkar MP, Bhutkar VM, Taware BG, Doijad V, Doddamani BR. Effect of suryanamaskar practice on cardio-respiratory fitness parameters: A Pilot Study. Al Ameen J Med Sci. 2008; 1: 126-9.

26. Charaka S, Sashtri Kashinath, Pt, Chaturvedi Gorakhnath., Dr.Ibid verse 15/4. Varanasi: Chaukhamba Bharti Academy; 2004. Chikitsasthana, 15/4; p. 452)

27. Das Nabanita, Choudhary Kuldeep, Kanika Goswami. Trikatu Churna in the Management of Hypothyroidism. International Journal of Ayurveda and Pharma Research. 2018; 6(4):71-74.

28. Dr. Nidhi Gupta, Dr. Kshiteeja Choudhary, \& Dr. Gopesh Mangal. (2020). Conceptual study on Partimarsha Nasya- A Review Article. Journal of Ayurveda and Integrated Medical Sciences, 5(04), 367-372.

29. Tanwar SR, Thakar AB, Ramteke R. Clinical evaluation of Nasya Karma in cervical spondylosis: Case series. Indian J Health Sci Biomed Res 2017; 10: $335-9$

30. Effect of Suryanamaskar on han healthy volunteers in http://statperson.com/journal/science and technology/Articl e/Volume12Issue2/12_2_5.pdf

31. Hedaoo MM, Bhole TP. Narrative review of guggulu formulations of Ayurveda reflecting their percentage of guggulu, pharmaceutics and pharmacology. IP Int J Comprehensive Adv Pharmacol 2020; 5(4):151-157.
Cite this article as:

Shantala Priyadarshini, Pannaga N terragundi, Pooja Gangadkar, Priyanka. Ayurvedic Successful Management of Nodular Episcleritis With Prolonged Period Follow Up- A Case Report. International Journal of Ayurveda and Pharma Research. 2021;9(9):74-79. https://doi.org/10.47070/ijapr.v9i9.2010

Source of support: Nil, Conflict of interest: None Declared
*Address for correspondence Dr. Shantala Priyadarshini Director \& Senior consultant, Dr. Vijay's Ayurveda Research $\&$ therapy centre, 1 st main, $18^{\text {th }}$ cross, Jayanagar. Mysore, Karnataka.

Email:

shantala301@gmail.com

Disclaimer: IJAPR is solely owned by Mahadev Publications - dedicated to publish quality research, while every effort has been taken to verify the accuracy of the content published in our Journal. IJAPR cannot accept any responsibility or liability for the articles content which are published. The views expressed in articles by our contributing authors are not necessarily those of IJAPR editor or editorial board members. 